\title{
Petite enfance et reconnaissance. ANALYSES À PARTIR de l'CEUVRe D’AXEL HONNETH
}

\author{
EARLY CHILDHOOD AND RECOGNITION. ANALYSIS ON THE \\ WORK OF AXEL HONNETH
}

\section{Primeira infânCIa E ReCONhecimento. AnÁlises a PARTIR DA OBRA DE AXEL HoNNETH}

\author{
Eric Plaisance \\ Doutorado em Doctorat d'Etat ès Lettres et Sciences Humaines, Université de Paris V \\ (René Descartes), U.P. V, França. Professeur émérite de l'Université Paris Descartes. \\ ORCID: https://orcid.org/o0oo-0002-7047-4I94 \\ erplaisance@gmail.com
}

\begin{abstract}
RéSUMÉ: Le concept de reconnaissance est central dans l'œuvre du philosophe et sociologue allemand Axel Honneth. Ce n'est pas la reconnaissance au sens de la perception des choses ou des êtres, mais la reconnaissance morale et intersubjective des personnes, ce qui est, selon Honneth, au cœur de la constitution du lien social. Le premier niveau de cette reconnaissance concerne les relations affectives et, tout d'abord, les relations entre le jeune enfant, sa mère et son environnement proche. Dans ce texte, je propose de prolonger et de compléter les analyses de Honneth sur les processus de reconnaissance de la petite enfance en distinguant plusieurs aspects: la reconnaissance de l'âge spécifique de la petite enfance au cours de l'histoire; la reconnaissance moderne du jeune enfant et même du bébé comme une "personne " et leur place dans les institutions telles que crèches, jardins d'enfants, écoles maternelles (en prenant l'exemple de la France après la seconde guerre mondiale) ; les processus de reconnaissance mutuelle des jeunes enfants (par exemple à travers les activités de coopération dans les jeux); la reconnaissance interactive des jeunes enfants avec déficiences et difficultés diverses dans la problématique internationale actuelle de l'éducation inclusive.
\end{abstract}

Mots CLEFS: Education Inclusive; Interaction; Lien Social; Petite Enfance; Reconnaissance.

ABstraCt: The concept of recognition is central to the work of the German philosopher and sociologist Axel Honneth. For him, recognition does not refer to the perception of things or beings, but to the moral and intersubjective recognition of people, which, according to Honneth, is at the heart of the social link foundation. The first level of 
this recognition concerns the emotional relationships and at the very beginning, the relationship between a young child, his mother and his immediate environment. I propose to extend and complete Honneth's analysis of early childhood recognition processes by distinguishing the following aspects: the recognition of the specific age of early childhood during the course of history; the modern recognition of the young child and even the baby as "a person" and their place in institutions such as kindergartens and daycares (using the example of France after the Second World War); the processes of mutual recognition of young children with each other (through cooperating play activities by example); the interactive recognition for young children with deficiencies and difficulties in the current international framework of inclusive education.

KeYwords: Inclusive Education; Interaction; Social Link; Early Childhood; Recognition.

Resumo: O conceito de reconhecimento é central na obra do filósofo e sociólogo alemão Axel Honneth. Não é o reconhecimento no sentido da percepção das coisas ou dos seres, mas o reconhecimento moral e intersubjetivo das pessoas, que segundo Honneth é o núcleo do laço social. O primeiro nível deste reconhecimento concerne às relaçóes afetivas e se inicia nas relaçóes entre a criança, a mãe e o meio ambiente circundante. Neste texto, eu me proponho aprofundar e completar as análises de Honneth sobre os processos de reconhecimento, distinguindo vários aspectos: o reconhecimento da idade específica da primeira infância no curso da história; o reconhecimento moderno da criança pequena e mesmo do bebê como uma "pessoa" e do seus lugares nas instituiçóes tais como creches, jardins de infância, escolas maternais (tomando o exemplo da França depois da segunda guerra mundial); os processos de reconhecimento mútuo entre essas mesmas crianças (por exemplo, por meio das atividades de cooperação nos jogos); o reconhecimento interativo dessas crianças com deficiências e com diversas dificuldades na problemática internacional, atual, da educação inclusiva.

Palavras-Chave: Educação Inclusiva; Interação; Laço Social; Primeira Infância; Reconhecimento.

\section{Introduction}

Les recherches sur la petite enfance ont longtemps été dominées par les approches médicales. La psychologie a ensuite introduit un autre regard sur le développement de l'enfant, un regard non limité aux questions d'hygiène et de soins corporels, mais prenant en compte ses capacités psychiques naissantes et ses modes de relation avec son entourage. Mais malgré d'assez nombreux bilans comparatifs internationaux sur les politiques d'accueil et d'éducation de la petite enfance dans différents pays, par exemple en Europe, malgré des enquêtes de terrain sur les institutions et les professionnels impliqués, les travaux sur la petite enfance restent encore lacunaires. Des déficits de connaissances subsistent sur les modes 
de socialisation du jeune enfant en dehors de sa propre famille, c'est-à-dire sur sa place dans différents dispositifs collectifs, sur ses interactions avec les adultes et avec ses pairs. Au-delà des descriptions nécessaires des réalités vécues par les enfants et les professionnels, on doit aussi s'interroger sur les cadres conceptuels qui permettent de mieux analyser et interpréter les constats qui ne suffisent jamais à eux seuls. C'est en fonction de ces préoccupations que je propose ici une relecture d'un certain nombre de travaux sur la petite enfance en sciences humaines et sociales, en utilisant le concept de reconnaissance qui est central dans l'œuvre d'Axel Honneth. J'espère ainsi contribuer à l'avancement des recherches en sociologie de la petite enfance.

\section{Le concept de reconnaissance chez Axel Honneth}

Le concept de reconnaissance est fondamental dans l'œuvre du philosophe et sociologue allemand Axel Honneth, né en 1949, qui est considéré comme l'un des grands représentants actuels de l'Ecole dite " de Francfort ". Sous ce dernier terme, on désigne un courant de pensée en Allemagne qui remonte aux années de l'entre deux guerres mondiales (création de l'Institut de Recherches Sociales à Francfort en 1923) et qui se revendique aux limites entre la sociologie et la philosophie, comme théorie critique alliant la théorie sociale, la perspective critique et la perspective émancipatrice. (DURAND-GASSELIN, 2012; ASSOUN, 1987)

Pour l'élaboration du concept de reconnaissance, Axel Honneth s'inspire de la philosophie de Hegel sur la vie éthique et sur la lutte pour la reconnaissance. Il précise que ce n'est pas la reconnaissance au sens de la perception des choses ou des êtres, mais la reconnaissance morale et intersubjective des personnes, qui " constitue en quelque sorte le coeur du social.» (HONNETH, 2006, p. I54) Ce n'est donc ni la connaissance, ni même la reconnaissance au sens de la perception ou de l'identification d'une chose ou d'un être déjà connu auparavant (par exemple, lorsque je reconnais le visage de quelqu'un) mais de la reconnaissance dans son sens moral, confirmant la valeur sociale reconnue à une personne dans une relation intersubjective. Parmi les nombreuses définitions de la reconnaissance proposées par Honneth dans ce sens précis, retenons celle-ci: 
[...] reconnaître quelqu'un signifie percevoir en lui des qualités qui nous incitent de manière intrinsèque à nous comporter non plus de manière égocentrique mais conformément aux intentions, besoins ou désirs de cette autre personne. Il est alors évident que l'attitude de reconnaissance doit présenter les traits d'une action morale parce qu'elle se laisse imprégner par la valeur d'autres personnes [...] (op.cit., p. 26I)

En partant de cette conception de la reconnaissance comme attribution de valeur à une personne ou à un groupe, Honneth (20I0, p. II6) distingue trois niveaux de reconnaissance ou trois sphères d'interaction, correspondant à un élargissement progressif des personnes concernées: les liens affectifs dans l'amour et l'amitié, la reconnaissance juridique dans le droit, l'adhésion à des valeurs communes dans la solidarité.

Ce qui retient actuellement mon attention est le premier niveau de cette reconnaissance, car elle vise le jeune enfant, voire le tout jeune enfant, c'est-à-dire le nourrisson. Pour préciser le sens de ce premier niveau, Honneth se réfère au courant théorique de la psychanalyse sur la « relation d'objet " et aux recherches empiriques développées par René Spitz, John Bowlby, ou encore Donald Winnicott. Selon Honneth, l'avantage de ces auteurs est qu'ils permettent de comprendre les premières relations intersubjectives du jeune enfant avec son entourage immédiat et, plus spécifiquement, sa mère. Ce sont des relations qui ne se limitent nullement à des satisfactions des besoins physiologiques élémentaires mais qui impliquent des soins de nature interactive et une attention à la personne du jeune enfant. Honneth retient chez Spitz que la privation de soins maternels (au sens du manque d'attention individualisée) cause de graves perturbations chez le nourrisson (dépression, marasme, pouvant aller jusqu'à la mort). Chez Winnicott, il retient l'idée d'une progression allant de la dépendance absolue du jeune enfant (symbiose avec la mère) à sa dépendance relative, et il insiste sur l'hypothèse originale de "l'objet transitionnel ", sphère de réalité intermédiaire entre l'enfant et le monde extérieur, qui lui permet d'accéder à une première forme d'autonomie et à une capacité d'être seul (HONNETH, 20IO, p. I2I et suiv.): «[...] l'amour nous est apparu comme l'expression affective d'une sollicitude qui résiste même à l'éloignement.» (op.cit. p. I44) 
De l'ensemble de ces références, Honneth tire la conclusion que la structure intra-psychique du sujet-enfant s'élabore comme " un processus d'intériorisation par lequel l'enfant assimile progressivement les schémas d'interaction qu'il doit apprendre dans la rencontre avec les personnes de référence successivement apparues: sa mère, son père, ses frères et soeurs et finalement aussi ses pairs.» (HONNETH, 20I3, p. 236)

Je propose de prolonger et de compléter les analyses de Honneth sur les processus de reconnaissance de la petite enfance en distinguant plusieurs aspects:

- la reconnaissance de l'âge spécifique de la petite enfance au cours de l'histoire, en insistant sur la période qui suit la seconde guerre mondiale en France où le petit enfant (y compris le nourrisson) est reconnu comme "une personne " dans ses interactions avec son entourage, ce qui va de pair avec la valorisation d'institutions collectives pour le jeune enfant (crèches et écoles maternelles).

- la reconnaissance mutuelle des jeunes enfants entre eux dans leurs relations intersubjectives.

- la reconnaissance des jeunes enfants avec déficiences et difficultés diverses dans la problématique internationale actuelle de l'éducation inclusive.

\section{La reconnaissance de la spécificité du jeune enfant au cours de l'histoire}

La représentation de la spécificité de l'enfance et de la petite enfance n'est pas une donnée spontanée et naturelle. Des variations importantes existent dans la différenciation de catégories d'âge entre les sociétés actuelles, et on relève encore des processus historiques de ce " découpage " des âges. Les travaux de Philippe Ariès sont des références majeures pour répondre à cette interrogation sur ce qu'il appelait "le sentiment » de l'enfance, c'est à dire sur la construction historique de la conscience des particularités de l'enfant, sa non-assimilation à un adulte en réduction. Dans son ouvrage célèbre sur l'enfant et la vie familiale sous l'Ancien Régime, Philippe Ariès (1960) a voulu trouver une origine à une perception nou- 
velle de l'enfant, en observant l'évolution des jeux et des vêtements utilisés par les enfants. Selon lui, le $17{ }^{\text {ème }}$ siècle aurait marqué une étape fondamentale vers cette conscience de la particularité enfantine qui se serait développé principalement dans les classes bourgeoises et dans la noblesse éclairée, et non dans les classes populaires de cette époque. En fonction des débats et des critiques qui ont suivi cette thèse, Ariès lui-même a nuancé ses analyses, en disant avoir trop cherché un point origine, un point zéro qui aurait vu émerger brusquement cette nouvelle conscience, alors que d'autres chercheurs ont montré que les témoignages d'affection à l'égard de l'enfant sont repérables, par exemple au Moyen Age. Mais ce sont bien ces travaux pionniers d'Ariès qui ont permis le développement de recherches sur l'histoire de l'enfance et amorcé des recherches portant plus spécifiquement sur la petite enfance. Ainsi, des historiens ont porté leur attention sur les tout premiers âges de la vie et même sur les pratiques anciennes de l'accouchement et aux soins au bébé nouveau-né (GELIS; LAGET; MOREL, 1978). D’autres auteurs ont étudié le développement de la scolarisation de la petite enfance. Jean-Noël Luc a traité de 'l'invention' du jeune enfant au XIXème siècle, en utilisant l'expression invention non dans le sens de créer un imaginaire mais, conformément aux orientations initiales d'Ariès, dans le sens d'une nouvelle attention aux successions des âges au sein de la petite enfance elle-même (LUC, I998). Il repère, au I9 $9^{\text {ème }}$ siècle, l'usage de la notion de 'seconde enfance', c'est à dire au-delà de l'âge de 2 ans, et corrélativement, le développement de la scolarisation à partir de cet âge jusqu'à 6 ans environ, avant l'âge de la scolarisation primaire. Ce sont d'abord les 'salles d'asile' à partir des années I820, qui étaient des salles d'hospitalité mais aussi des salles pour commencer une éducation collective et même des éléments d'instruction, puis, dans les années I880, ce sont les écoles maternelles qui se situent dans le cadre de politiques républicaines en faveur de l'école primaire laïque. La reconnaissance du jeune enfant trouve un ardent défenseur dans la personne de l'inspectrice générale des écoles maternelles, Pauline Kergomard, qui déclarait que le jeune enfant n'est pas «matière scolaire» et que le jeu c'est le «travail de l'enfant.» (PLAISANCE, I996)

De son côté, l'histoire des crèches a été de mieux en mieux documentée. Cette idée d'un accueil collectif des enfants en dessous de l'âge de 2 ans dans les institutions spécifiques est une autre nouveauté au $19^{\text {ème }}$ 
siècle en France. Elle succède au temps des nourrices, mais n'élimine pas non plus le recours des parents à des femmes chargées de s'occuper de leurs enfants aussi bien en milieu populaire que dans des milieux socialement privilégiés. Dans le cas des familles populaires, les très jeunes enfants étaient menés souvent loin du domicile (avec un système de transporteurs dits 'meneurs' ou 'meneuses'), et dans les familles disposant de ressources, les nourrices étaient à domicile. Mais le travail des femmes de milieu populaire dans les nouvelles industries et la nécessité de faire garder les enfants dès leur plus jeune âge a obligé à développer des modes de garde plus systématiquement organisés. Les crèches apparaissent un peu plus tard que les salles d'asile mentionnées précédemment, soit dans les années i 840 sous l'impulsion du 'catholicisme social', avec une forte empreinte religieuse catholique mais soucieuse d'améliorer les conditions de vie des familles ouvrières (KNIBIEHLER, 2004). Les crèches sont progressivement créées et développées avec un souci complémentaire à celui de la garde: le souci de moraliser les classes populaires, puis à la fin du I ${ }^{\text {ème }}$ siècle et surtout au début du zoe siècle, le souci de développer l'hygiène, en améliorant la santé physique du jeune enfant et en diffusant les 'bonnes manières' dans les familles par les règles de puériculture. (BOUVE, 200I)

Cette phase de la médicalisation au début du $20{ }^{\text {ème }}$ siècle en France (qui se manifeste clairement dans les écoles maternelles et dans les crèches) a donc non seulement un objectif sanitaire de réduction de la mortalité infantile mais aussi un objectif de moralisation sociale envers les classes populaires. Corrélativement, la reconnaissance du jeune enfant est alors soumise à des idéologies religieuses et hygiénistes qui font de l'enfant lui-même un instrument de modification des moeurs. Sauf chez quelques auteurs pédagogues (comme Pauline Kergomard, déjà citée à propos de l'école maternelle), la spécificité du jeune enfant se limite généralement à la nécessité d'attention à sa condition physique et à l'application de soins hygiénistes.

\section{Reconnaissance de la petite enfance et institutions après la deuxième guerre mondiale en France}

Une autre phase de développement des institutions pour la petite enfance se situe après la deuxième guerre mondiale. Elle révèle de nou- 
velles représentations du jeune enfant. Si l'on suit les précisions conceptuelles apportées par Axel Honneth, la reconnaissance n'est plus seulement la reconnaissance du caractère spécifique de la petite enfance, comme précédemment (c'est-à-dire perçue comme distincte des autres âges), elle devient aussi la reconnaissance du petit enfant en tant que personne et dans les relations intersubjectives. Or, ce processus ne semble clairement engagé qu'après la seconde guerre mondiale, en même temps que s'opère un processus continu de valorisation des enfants et de transformation des rapports éducatifs, ce que Alain Renaut (2003) résume par l'expression « libération des enfants ».

Les années qui suivent la fin de la seconde guerre mondiale sont appelées les années de La Libération, avec ce que cela comporte d'emphase mais aussi de volonté de reconstruire le pays sur de nouvelles bases dans les domaines les plus divers (politique, santé, éducation etc.). Cependant, pour les soins et l'éducation de la petite enfance, les options manifestées restent contradictoires. D'un côté, les milieux les plus conservateurs se présentent pour la défense de la famille traditionnelle et donc en faveur de la garde des enfants par la mère. Ils expriment les plus grandes réticences à l'égard des modalités de garde collective, telles que les crèches. Certains peuvent appuyer leur argumentation sur la diffusion nouvelle de connaissances sur les cas dits 'd'hospitalisme', c'est à dire sur des situations d'enfants en carence de soins maternels et de liens affectifs et pouvant développer des pathologies allant sur la mort: ce sont les cas de dépression et de 'marasme' décrits dans les travaux célèbres de René Spitz et rapportés par de nombreux auteurs, par exemple par Myriam David (20I4, p. I57) mais aussi Axel Honneth lui-même. Mais, d'un autre côté, des données différentes mettent en valeur les capacités relationnelles précoces des jeunes enfants, y compris en dehors de leur entourage familial immédiat. Des positions se sont de plus en plus affirmées en faveur d'institutions favorisant le développement de ces capacités, principalement les crèches et les écoles maternelles, dans une période où la natalité en forte progression obligeait à concevoir des solutions de garde. Un exemple institutionnel étranger souvent cité chez les auteurs français (et visité par certains d'entre eux) révèle même les capacités des jeunes enfants à se lier entre eux et à être autonomes, sous la condition que les adultes sachent organiser un environnement favorable: c'est l'institut Loczy à Budapest en Hongrie, va- 
lorisant le mouvement libre de l'enfant, pris comme modèle d'institution bienveillante au développement, sur le plan à la fois moteur et psychique. (DAVID; APPEL, I973; DAVID, 2014, p. 250; GOLSE, 2003, p. 66)

Plus généralement, comment caractériser les transformations des représentations des jeunes enfants dans cette période qui suit la seconde guerre mondiale? Ou encore, quelle nouvelle définition de la prime enfance devient dominante ? Le trait essentiel de cette évolution est la "découverte de la prime enfance comme objet pédagogique »(CHAMBOREDON; PREVOT, 1973), c'est-à-dire une valorisation culturelle et psychologique du jeune enfant et une moindre importance apportée aux soins hygiéniques et physiologiques, contrairement aux représentations traditionnelles. La nécessité de tels soins n'est pas niée mais elle recule vers les âges de plus en plus jeunes, vers le nourrisson. En d'autres termes, le jeune enfant, et même le très jeune enfant, n'est plus seulement considéré comme une bouche à nourrir ou un corps à soigner, mais avant tout comme un être en développement. La focale se déplace vers une vision du jeune enfant dans sa dimension psychologique et culturelle. Un trait caractéristique de cette évolution est la qualification du bébé lui-même comme 'une personne'. Le succès du film Le bébé est une personne, en 1984, puis de l'ouvrage en 1985 de Bernard Martino avec ce même titre, est emblématique de ces transformations radicales de représentations. C'est un autre pas en avant vers une reconnaissance qui ne se limite plus à concevoir que le jeune enfant est spécifique et différent des âges plus avancés, car cette reconnaissance moderne vise maintenant à lui attribuer des caractéristiques de personnalité qui lui sont propres, des particularités qui le distinguent d'un autre enfant. La diffusion médiatique de ce thème avec une forte référence à des travaux de psychologues ou de psychanalystes a beaucoup fait pour son succès. On constate, dans ces conditions, des transferts nombreux entre la littérature savante et la vulgarisation en direction du grand public, particulièrement en direction du public féminin par les revues qui lui sont destinées: «[...] sous l'action de la diffusion médiatique des idées sur le petit enfant, celuici est de plus en plus perçu comme un être 'à part entière', sur lequel un investissement s'est effectué.» (NEYRAND, 2000, p. I4)

Jean-Claude Chamboredon et Jean Prévot (I973, p. 3I2) soulignent, eux aussi, ce rôle de la médiatisation: «[...] la psychologie a conduit à la découverte de la prime enfance en persuadant de l'importance de cette 
période pour la constitution de la personnalité et, surtout, pour la formation de l'intelligence.» Ces deux auteurs ont surtout insisté sur le fait que l'école maternelle, après la seconde guerre mondiale, est tributaire de cette nouvelle définition de la petite enfance: il y a «inscription dans les jeux, dans le matériel, dans l'organisation entière de l'espace (matériel, disposition architecturale) de cette définition nouvelle [...]» à tel point que cette école pourrait être considérée comme "une sorte de grand jouet éducatif.» (op.cit., p. 32I) Ils précisent aussi que les différentes classes sociales qui utilisent l'école maternelle n'ont pas le même rapport à cette nouvelle valorisation du jeune enfant et des activités ludiques: les classes populaires se situent plutôt du côté des demandes utilitaires de garde, alors que les classes moyennes et supérieures présentent des attentes 'cultivées' envers cette école du jeu et du développement du jeune enfant.

Ce type d'analyse sociologique a été prolongé par une enquête portant sur les nouveaux usages de la maternelle à Paris par les différentes classes sociales entre 1945 et I980 (PLAISANCE, I986). Alors que la fréquentation traditionnelle est, en début de période, une fréquentation populaire, la fréquentation s'étend ensuite aux classes moyennes et même aux classes supérieures: l'école maternelle voit sa fréquentation généralisée à l'ensemble des parents, quelle que soient leurs appartenances sociales. ${ }^{2}$ Parallèlement, l'étude détaillée de l'évolution des modèles pédagogiques pratiqués en écoles maternelles dans la même période montre la progression sensible d'un modèle appelé 'expressif': c'est-à-dire la priorité accordée aux capacités expressives de l'enfant, à son originalité personnelle, par exemple manifestée dans les activités de jeu, le dessin etc. (PLAISANCE, I986)

Ces nouvelles valorisations de l'enfant, qui contrastent fortement avec les représentations traditionnelles, ne sont pas spécifiques aux écoles maternelles dans leurs évolutions après la seconde guerre mondiale. On constate les mêmes tendances dans les crèches et, par conséquent, pour les enfants plus jeunes. Malgré les prescriptions médicales et para médicales qui ont longtemps dominé les discours et les pratiques, les orientations de nature psychologique prennent une nouvelle place dans les années 60: il s'agit maintenant d'être attentif non seulement aux besoins corporels mais encore au développement total du jeune enfant, comprenant ses capacités psychologiques. Il y a donc « une revalorisation psychologique 
de la crèche collective qui elle-même se trouve confortée et valorisée par la nouvelle qualité sociale d'une partie de la clientèle, ouverte au discours psychologique.» (BOUVE, 200I, p. 6I) ${ }^{3}$ Qu'il s'agisse des écoles maternelles ou des crèches après la seconde guerre mondiale, on constate donc, dans les deux cas, une nouvelle reconnaissance de la petite enfance, soutenue par la vulgarisation de la psychologie moderne, en des termes de valorisation de la personne en tant que telle et de ses multiples capacités expressives.

\section{Les jeunes enfants entre eux}

Dans les analyses précédentes, une dimension de la reconnaissance n'a pas été abordée et elle n'est pas non plus traitée comme telle chez Honneth. C'est la dimension sociétale du jeune enfant, celle de la relation avec l'autre enfant, une dimension souvent ignorée, car recouverte par la conception erronée de l'enfant 'égocentrique', replié sur lui-même et incapable de coopération avec ses pairs. Or, les processus de reconnaissance mutuelle des jeunes enfants à travers diverses activités sont l'objet de recherches relativement nombreuses qui invalident ce type de préjugé.

A partir des années 1970, les travaux pionniers sur les phénomènes d'interaction sociale précoce émanent de psychologues. Une équipe française du Centre de Recherche de l'Éducation Spécialisée et de l'Adaptation Scolaire (CRESAS) a montré toute la richesse des relations entre enfants âgés de moins de deux ans en crèche (STAMBAK, I983). Ces psychologues ont effectué des observations de jeunes enfants non dans des situations artificielles de laboratoire, mais en milieux 'naturels', pour repérer les types de relation que les enfants établissent entre eux. Ils constatent que les bébés entre eux sont capables de découvrir, de jouer, d'inventer ensemble. Des situations diverses sont captées par des films et systématiquement explorées ensuite dans tous leurs détails: jeux moteurs, explorations d'objets, jeux de faire semblant. Quelles que soient ces variétés, deux dimensions des capacités enfantines apparaissent étroitement liées: celle des capacités cognitives et celle des capacités affectives. Dans l'exemple de jeux libres avec de grandes boites en carton, «l'élan vers autrui se manifeste aussi bien par des regards, des mimiques, des postures, des attitudes, des vocalises 
que par le fait de se rapprocher les uns des autres.» (op.cit., p. 27) Les jeux de 'cache-cache' révèlent de manière très caractéristique la propension sociale des jeunes enfants, car de tels jeux impliquent non seulement d'être à plusieurs mais aussi une indispensable entente sur le sens du jeu: se cacher pour être découvert! De même, les jeux de faire semblant (par exemple, faire semblant de donner à boire ou à manger à un partenaire) nécessitent une coopération entre les partenaires. C'est donc une vie sociale précoce qui est observée chez des jeunes enfants de moins de deux ans qui ne communiquent pas encore verbalement (sinon par vocalises) et qui restent encore très maladroits pour se déplacer: "[...] nous découvrons des êtres sociables, attentifs les uns aux autres, désireux de faire des choses en commun, provoquant et 'jaugeant' les réactions d'autrui.» (op.cit. p. I82)

La reconnaissance de l'autre dans les interactions entre jeunes enfants a fait l'objet de recherches plus récentes en sociologie de l'enfance. Cette orientation de recherche, développée principalement à partir des années I990 considère l'enfance comme une composante structurale de la société, et non comme une phase de développement psychophysiologique ou comme un simple moment précurseur de l'adulte. Ce qui mène à un regard nouveau sur les cultures enfantines, sur les phénomènes de transmission de valeurs, de représentations et de pratiques dans le milieu enfantin lui-même (SIROTA, 2006; SARMENTO, 2008; DELALANDE, 2009; REIS, 20I5). ${ }^{4}$ Les activités de transmission entre pairs, c'est à dire les enfants entre eux, retiennent l'attention des chercheurs car elles révèlent les spécificités des interactions entre enfants et de leurs socialisations et, plus encore, elles créditent les enfants d'un statut d'acteurs sociaux. Ici encore, le jeu peut être présenté comme l'aspect essentiel de la culture enfantine. Ainsi, l'observation des activités des enfants en cour de récréation, qui parait comme le 'théâtre d'une microsociété', montre la créativité des jeunes enfants pour inventer des jeux qui impliquent de les partager à plusieurs. (DELALANDE, 200I). Lobservation, par le même auteur, du jeu du 'sable doux' montre les capacités des enfants à utiliser un matériau banal, présent dans les bacs à sable des cours de récréation, pour fabriquer un sable purifié de ses cailloux et en faire un sable fin, objet de convoitises et d'échanges subtils entre enfants. De plus, le sable précieusement sélectionné est comparé symboliquement à des ingrédients culinaires (du miel ou du chocolat selon la couleur du sable). "L'omniprésence de 
l'échange dans le jeu de sable doux s'opère non seulement entre deux enfants mais par une pression qui tient à valoriser le respect de la 'loi des pairs' dont fait partie ce principe d'échange.» (DANIC et al., 2006, p. I32; voir aussi DELALANDE, 200I)

De tels partages d'activités et ces instaurations de rituels enfantins ne relèvent pas de processus passifs d'imitation mais manifestent plus profondément des reconnaissances actives entre pairs. Il s'agit de phénomènes de socialisation horizontale entre pairs dont l'observation précise complète la vision trop étroite de la socialisation héritée de Durkheim comme transmission verticale de la génération adulte vers la génération plus jeune. En réalité, les enfants, y compris les enfants très jeunes, savent très pertinemment se glisser dans les interstices des activités permises par les adultes pour développer leurs propres activités, y manifester leurs intérêts et développer leurs propres interactions. (GARNIER et al., 20I6)

\section{Reconnaissance et éducation inclusive}

Le processus de reconnaissance des jeunes enfants avec handicaps, besoins particuliers ou difficultés diverses, est au centre de la problématique internationale actuelle de l'éducation inclusive. Contrairement à la problématique ancienne de l'éducation spéciale qui impliquait une séparation entre le secteur éducatif commun et le secteur 'spécial', l'éducation inclusive implique une reconnaissance pleine et entière de tout enfant au sein de l'institution scolaire et donc une appartenance au milieu commun. C'est une reconnaissance de la diversité et de sa valeur au sein des institutions éducatives.

Laccueil en crèches, en jardins d'enfants ou en écoles maternelles permet, en principe, une plus grande ouverture à la diversité des comportements. Ce sont des institutions qui sont généralement plus inclusives. Le processus de reconnaissance de la diversité s’y trouve plus accessible et plus développé, du fait de l'absence de programmes rigides d'enseignement, comme on en trouve dans l'enseignement primaire ou secondaire, mais aussi du fait que les jeunes enfants sont considérés par les éducateurs et les éducatrices comme des êtres en évolution et susceptibles de changements rapides. 
La reconnaissance de cette diversité peut d'abord être analysée sous l'angle institutionnel et en rapport avec le travail des professionnels. Une enquête comparative européenne sur l'éducation inclusive dès la petite enfance avait pour objectif de fournir des analyses sur les pratiques existantes en montrant les conditions favorables aux pratiques inclusives (en français: KRON; PLAISANCE 20I2; en portugais: KRON; SERRANO; LIMA ALFONSO, 20I4). Les questions générales étaient les suivantes: comment faciliter la coéducation et l'inclusion d'enfants qui présentent des «besoins particuliers»; comment pratiquer une éducation en commun, en supprimant les barrières et les discriminations traditionnelles?

Dans le bilan de ce travail international, on constate des différences entre les pays, dans leurs institutions, leurs modes d'organisation, leurs modèles pédagogiques, les statuts des professionnels. Sous cet aspect, on ne relève pas d'uniformité. ${ }^{6}$ Mais des convergences fondamentales subsistent sur l'accueil de la diversité dans ses différentes dimensions: il s'agit non seulement de la diversité des enfants, incluant des enfants avec 'besoins particuliers', mais aussi de la diversité des familles, par exemple des familles issues de l'immigration, dans le cadre des sociétés européennes de plus en plus hétérogènes. Du point de vue des pratiques observées, les activités collectives, favorisant le lien social, étaient considérées comme essentielles car elles formaient le cadre de vie quotidienne destiné à tous. On peut alors considérer que le processus de reconnaissance des enfants avec besoins particuliers impliquait aussi bien la dimension collective (reconnaissance dans les groupes) et la dimension individuelle (reconnaissance dans les actions pédagogiques différenciées et dans les aides particulières).

Dans le cadre général de cette recherche, une enquête approfondie de type ethnographique a été menée dans un jardin d'enfants inclusif à Paris (avec financement public) où le projet institutionnel est de recevoir I/3 d'enfants reconnus officiellement comme ayant des besoins particuliers (et parfois avec des incapacités graves) et $2 / 3$ d'enfants 'valides'. L'observation d'un épisode particulier a permis de repérer des conditions favorables à la reconnaissance pratique d'une petite fille présentant des difficultés psychiques de type psychotique (comportement très perturbé, difficultés d'attention, activités isolées et bruyantes à l'écart des groupes etc.). 
Au cours d'un atelier de cuisine dirigé par une éducatrice avec un petit groupe de six enfants âgés d'environ 5 ans, il s'agissait de confectionner un gâteau en commençant par mélanger la farine. Or, la petite fille qui venait de rejoindre le groupe s'est mise à souffler fortement sur la farine, ce qui a provoqué un nuage de farine au-dessus de la table. La réaction de l'éducatrice fut la suivante: «Oh! Regardez. Elle vient de souffler sur la farine et ça fait de la poussière ! C'est tellement fin que ça fait de la poussière!» C'est un moment qui suscite beaucoup d'intérêt de la part des autres enfants. Un garçon dit: «je peux essayer?» L'éducatrice incite les autres enfants à souffler eux aussi sur la farine: «Essaye, vas-y! Regardez ! Souffle, souffle fort! " Finalement tous les enfants suivent l'exemple donné par la petite file. (PLAISANCE, apud KUPFER, 20I7, p. 244-245)

Ce qui est remarquable dans cet épisode est que l'éducatrice accepte que la petite fille ne se comporte pas comme les autres. Elle ne 'redresse' pas le comportements déviant (souffler sur la farine) et cherche au contraire à l'utiliser pour l'adapter à tous, incitant même les autres enfants à constater l'intérêt de souffler sur la farine. Elle encourage une activité partagée et les interactions entre enfants. Cela manifeste une flexibilité 'pédagogique' de la part de la professionnelle qui sait utiliser l'inattendu pour inventer des pratiques profitables à tous. Autrement dit, la reconnaissance et l'acceptation de la particularité de la petite fille est aussi liée à une reconnaissance interactive dans l'ensemble du groupe d'enfants: malgré ses difficultés de comportement, la petite fille est reconnue comme membre du groupe.

Ce thème de la reconnaissance interactive entre enfants 'différents', c'est à dire entre enfants valides et enfants avec besoins particuliers, est un champ de recherche nouveau et encore trop peu développé, alors que les parents et les professionnels s'interrogent sur la coexistence possible d'enfants différents dans des espaces communs. Parmi les travaux qui portent sur cette question (en plus de l'enquête internationale ci-dessus), des observations effectuées en écoles maternelles et en crèches en France sont riches de données de réflexion à la fois théoriques et pratiques.

Par exemple, deux chercheurs ont analysé des parcours d'inclusion, en effectuant des portraits d'enfants avec besoins particuliers, selon une méthodologie d'enquête qualitative (BEDOIN; JANNER-RAIMONDI, 20I6; 20I7). La grande originalité méthodologique de ces travaux est d'avoir cherché à recueillir les points de vue subjectifs des enfants eux- 
mêmes, en utilisant des images ou des photographies d'école maternelle. L'aspect encore plus original est d'avoir permis aux jeunes enfants (avec besoins particuliers ou non) d'utiliser eux-mêmes un appareil photo pour prendre les photos dans l'école. Ensuite les chercheurs demandaient aux enfants de commenter leurs photos pour connaître leurs représentations de la vie quotidienne dans leur école maternelle.

Les résultats qui retiennent mon attention concernent surtout les relations entre enfants 'différents'. Dans des groupes de parole (focus groups), les enfants âgés de 4 à 6 ans se sont exprimés sur leur vie quotidienne à l'école où ils côtoyaient des camarades avec besoins particuliers (camarades avec syndrome de Down ou avec autisme ou encore avec déficience visuelle). Les questions spontanément abordées par les enfants ne concernent pas directement la déficience d'un autre enfant mais la socialisation, c'est à dire les relations et les comportements. Par exemple, ils disent: «il ne peut pas courir très vite»; «elle pleure quand elle tombe»; «il ne va pas tout seul dans le couloir.» Les chercheurs commentent ainsi: «Les enfants de moins de 6 ans ont un discours adapté et différencié en fonction de la situation de handicap vécue par leur pair (...) ils sont capables de repérer et d'exprimer avec pertinence le point de vue d'un autre qu'eux, ici celui de l'enfant en situation de handicap.» (BEDOIN; JANNER-RAIMONDI, 20I6, p. I23 et p. 238) Dans une interprétation plus générale de ces données, les mêmes auteurs utilisent la notion d'empathie dans les termes suivants: «cette capacité d'empathie révèle un sujet capable de prendre en compte les autres (...), met en scène une intersubjectivité entendue comme une relation des sujets entre eux.» (op.cit., p. 220)

En utilisant ici la notion d'empathie à propos des réactions des jeunes enfants, les auteurs font aussi référence au concept de reconnaissance chez Axel Honneth. Les deux concepts sont considérés comme très proches et leur dimension éthique est soulignée dans la discussion, c'est à dire une dimension qui implique la reconnaissance de l'autre et la réciprocité. Mais les auteurs font une distinction importante entre sympathie et empathie. La simple sympathie se limite à une relation superficielle de partage ou même de fusion de sentiments avec l'autre, alors que l'empathie, déjà présente chez les jeunes enfants, manifeste une perception des réactions des autres et de leurs éventuelles difficultés (par exemple, marcher, aller seul...). Les jeunes enfants ont déjà la capacité 
de se mettre mentalement à la place de l'autre, ce qui nécessite non seulement des capacités émotives mais aussi des capacités cognitives pour comprendre l'autre. (op.cit., p. 213)

D'autres enquêtes de terrain montrent le rapport entre ces réactions «empathiques» des jeunes enfants avec le type de pédagogie pratiquée dans les classes d'écoles maternelles. A vrai dire, ce n'est pas la pédagogie dans le sens de la transmission des savoirs et des apprentissages, mais plutôt dans le sens la communication de valeurs au sein d'un groupeclasse et dans la constitution du lien social. Les professeurs favorables à l'accueil d'enfants avec difficultés ou avec besoins particuliers dans leur classe pensent que la priorité est celle de l'attention à chaque enfant et non la discipline. Au contraire, les professeurs réticents ou défavorables à cet accueil attachent plus d'importance à la discipline en classe. Le 'style pédagogique' est donc une variable importante dans l'acceptation des différences et influe sur les réactions des enfants eux-mêmes (THOUROUDE, 1999). C'est une donnée importante confirmée dans une enquête comparative France-Allemagne sur les interactions entre enfants plus âgés avec ou sans handicap (entre 8 et I 2 ans d'âge). L'auteur constate des phénomènes de solidarité plus élaborés que pour des enfants plus jeunes, par exemple dans des écoles ou des classes inclusives où la pédagogie est fondée sur l'entraide et la collaboration entre enfants. L'auteur observe ainsi la place d'un enfant comme "délégué» pour l'aide et l'entraide auprès d'un autre enfant en difficulté. (SCHNEIDER apud DELALANDE, 2009, p. 94-I09)

\section{Eléments de conclusion}

Dans cet article, j'ai appliqué à la petite enfance le concept de reconnaissance, élaboré par Axel Honneth, en essayant de distinguer plusieurs aspects du concept. En adoptant ce 'fil rouge' au long de notre texte, j'ai fait le pari que la reconnaissance pouvait être un outil pertinent pour comprendre l'histoire de la représentation moderne du jeune enfant et de sa place dans les institutions, pour analyser les interrelations entre enfants, en nous référant aussi aux nouvelles approches de la sociologie de l'enfance. Finalement, j’ai 
présenté et discuté les orientations actuelles de l'éducation inclusive en prenant plusieurs exemples d'enquêtes de terrain sur la petite enfance.

Cette reconnaissance moderne se heurte pourtant à des obstacles face auxquels une attention critique doit être développée. Les diversités culturelles et linguistiques qui sont les réalités du monde contemporain, où les populations immigrantes sont nombreuses, sont confrontées aux préjugés et aux discriminations. Et le poids des normes sociales dominantes concerne très directement la petite enfance et entrave son plein développement. Ainsi, des orientations contestables sont souvent formulées en faveur d'une prévention mal comprise en termes de dépistages précoces de perturbations infantiles, sans que des précautions élémentaires soient prises pour éviter des regards dévalorisants et des mesures stigmatisantes. Tout un travail quotidien sur des approches non normalisantes de la petite enfance et des familles (BROUGERE; VANDENBROECK, 2007) est donc nécessaire pour ouvrir encore plus largement le domaine de la reconnaissance.

\section{Notes}

I Le philosophe Paul Ricoeur (2004, p. 383) distingue la reconnaissance comme identification, la reconnaissance de soi-même, enfin la reconnaissance mutuelle qu'il définit ainsi: «Être reconnu, si cela arrive jamais, serait pour chacun recevoir l'assurance plénière de son identité à la faveur de la reconnaissance par autrui de son empire de capacités.»

2 Cette généralisation de la fréquentation aboutit aujourd'hui à des taux spectaculaires: Ioo \% des enfants de 3 ans, 4 ans et 5 ans fréquentent l'école maternelle (publique ou privée).

3 L'introduction de psychologues professionnels dans les crèches françaises débute par une expérience menée en 1953 dans les services de la protection maternelle et infantile de Paris. Ensuite, il y a installation d'un service de psychologie dans les crèches et les consultations de nourrissons, avec une valorisation de l'évaluation psycho motrice du développement des jeunes enfants. (BOUVE, 200I, p. 59)

4 Selon Manuel Sarmento (apud Sirota, 2006, p. 3I 4): «[...] les cultures de l'enfance possèdent, avant tout, des dimensions relationnelles. Elles se constituent dans les interactions entre pairs et entre les enfants et les adultes: se structurent dans ces relations, des formes et des contenus de représentations distinctes."

5 Les universités étaient les suivantes: université de Siegen (Allemagne), université du Minho à Braga (Portugal), université Mälardalen/Västeras (Suède), université Eötvös Lorànd à Budapest (Hongrie), université Paris Descartes à Paris (France). Les institutions pour la petite enfance, en général pour des enfants âgés de 3 à 6 ans, étaient elles-mêmes diverses: kindergarten, jardins d'enfants, pré-écoles, écoles maternelles etc.

6 A titre d'exemples: en Suède, le modèle pédagogique est plutôt familial, y compris dans l'organisation de l'espace; en Allemagne, le modèle est centré sur le jeu, venant de l'inspiration de Froebel; en France le modèle est plus scolairement structuré, y compris pour les enfants jeunes. 


\section{Bibliographie}

ASSOUN, Laurent. L'école de Francfort. Paris: PUF, 1987.

BEDOIN, Diane ; JANNER-RAIMONDI, Martine. Petite enfance et handicap. Famille, crèche, maternelle. Grenoble: Presses Universitaires de Grenoble, 2016.

Parcours d'inclusion en maternelle. Portraits de jeunes en situation de handicap. Nîmes: Champ Social Éditions, 2017.

BOUVE, Catherine. Les crèches collectives: usagers et représentations sociales. Contribution à une sociologie de la petite enfance. Paris: L'Harmattan, 200I.

BROUGÈRE, Gilles; VANDENBROECK, Michel (org). Repenser l'éducation des jeunes enfants. Bruxelles: P.I.E. Peter Lang, 2007.

GARNIER, Pascale ; BROUGERE, Gilles ; RAYNA, Sylvie ; RUPIN, Pablo. A 2 ans, vivre dans un collectif d'enfants. Crèche, école maternelle, classe passerelle, jardin maternel. Toulouse: Editions Érès, 2016.

CHAMBOREDON, Jean-Claude; PREVOT, Jean. Le «métier d'enfant». Définition sociale de la prime enfance et fonctions différentielles de l'école maternelle. Revue Française de Sociologie, vol. XIV, n.3, juillet-septembre 1973, p. 295-316.

DANIC, Isabelle; DELALANDE, Julie; RAYOU, Patrick. Enquêter auprès d'enfants et de jeunes. Objets, méthodes et terrains de recherche en sciences sociales. Rennes: Presses Universitaires de Rennes, 2006.

DAVID, Myriam. Prendre soin de l'enfance. Toulouse: Editions Érès. 2014.

Scarabée, I973. ; APPEL, Geneviève. Loczy ou le maternage insolite. Paris: Editions du

DELALANDE, Julie. La cour de récréation. Contribution à une anthropologie de l'enfance. Rennes: Presses Universitaires de Rennes, 200I.

.(Org). Des enfants entre eux. Des jeux, des règles, des secrets. Paris: Editions Autrement, 2009.

DURAND-GASSELIN, Jean-Marc. L'école de Francfort. Paris: Gallimard. 2012.

GELIS, Jacques; LAGET, Mireille; MOREL, Marie-France. Entrer dans la vie.

Naissances et enfances dans la France traditionnelle. Paris: Gallimard/Julliard, 1978.

GOLSE, Bernard. Sobre a psicoterapia pais-bebê: narrativizado, filiaçấo e transmissão. Sao Paulo: Casa do Psicologo. 2003.

HONNETH, Axel. La société du mépris. Vers une nouvelle théorie critique. Paris: La Découverte, 2006. 
allemand, I992)

La lutte pour la reconnaissance. Paris: Editions du Cerf, 2010 (Original en Un monde de déchirements. Théorie critique, psychanalyse, sociologie. Paris:

La Découverte, 2013.

KNIBIEHLER, Yvonne. Modes de garde: permanences et changements à travers l'histoire. Spirale, n. 30, p. I5-22, 2004.

KRON, Maria ; PLAISANCE, Eric (org). Grandir ensemble. L'éducation inclusive dès la petite enfance. Suresnes: Institut National Supérieur de Formation et de Recherche pour l'Education des Jeunes Handicapés et les Enseignements Adaptés (INSHEA), 2012 .

KRON, Maria; SERRANO, Ana Maria; LIMA AFONSO, Joana (org). Crescendo juntos. Passos para a inclusão na educação da infância. Porto: Porto Editora, 2014.

LUC Jean-Noël. L'invention du jeune enfant au XIXe siècle. De la salle d'asile à l'école maternelle. Paris: Belin, I998.

MARTINO, Bernard. Le bébé est une personne. Paris: Balland, I985.

NEYRAND, Gérard. L'enfant, la mère et la question du père. Un bilan critique de l'évolution des savoirs sur la petite enfance. Paris: Presses Universitaires de France, 2000.

PLAISANCE, Eric. Para uma Sociologia da pequena infância. Educação e Sociedade, Campinas, vol. 25, n. 86, 2004, p. 22I-24I.

Politicas para pequena infância e a educação inclusiva: uma contribução para a Sociologia da pequena infância. In: REIS, Magali dos; OGG GOMES, Lisandra (org), Infância: sociologia e sociedade. São Paulo: Levana,20I5, p. 2 I- 40.

. Quais são as práticas inclusivas de educação no mundo contemporâneo? Análises críticas e perspectivas de ação. In: KUPFER, Maria Cristina; PATTO, Maria Helena Souza; VOLTOLINI, Rinaldo (org). Práticas inclusivas em escolas transformadoras: acolhendo o aluno-sujeito. São Paulo: Editora Escuta, 20I7, p. 229-246.

Pauline Kergomard et l'école maternelle. Paris: Presses Universitaires de France, 1996.

. L'enfant, la maternelle, la société. Paris: Presses Universitaires de France, I986.

REIS, Magali dos; OGG GOMES, Lisandra (org). Infância: sociologia e sociedade. São Paulo: Levana, 2015.

RENAUT Alain. La libération des enfants. Contribution philosophique à une histoire de l'enfance. Paris: Bayard/Calmann Lévy, 2002.

RICOEUR Paul. Parcours de la reconnaissance. Paris: Stock-Gallimard, 2004. 
SARMENTO, Manuel Jacinto. Sociologia da Infância: Correntes e Confluências. In: SARMENTO, Manuel Jacinto; GOUVÊA, Maria Cristina Soares de (org). Estudos da Infância: educação e práticas sociais. Petrópolis: Vozes, 2008, p. I7-39.

SIROTA, Régine. Eléments pour une sociologie de l'enfance. Rennes: Presses Universitaires de Rennes, 2006.

SCHNEIDER, Cornelia. Solidarité enfantine et intégration scolaire de enfants en situation de handicap. In: DELALANDE, Julie (org). La cour de récréation. Contribution à une anthropologie de l'enfance. Rennes: Presses Universitaires de Rennes, 2009, p. 94-109.

STAMBAK Mira (Org). Les bébés entre eux. Découvrir, jouer, inventer ensemble. Paris: PUF, I983

THOLLON-BEHAR, Marie-Paule (Org). Accueillir l'enfant entre 2 et 3 ans. Toulouse: Editions Érès, 2017.

THOUROUDE, Laurence. Tolérance pédagogique et intégration d'enfants handicapés à l'école maternelle. La nouvelle revue de l'adaptation et de la scolarisation. Suresnes: n.8, I999, p. II3-I29.

Recebido em 25 mai. 2019 / Aprovado em 4 set. 2019

\section{Para referenciar este texto:}

PLAISANCE, E. Petite enfance et reconnaissance. Analyses à partir de l'œuvre d'Axel Honneth. EccoS - Revista Científica, São Paulo, n. 50, eI3998, jul./set. 2019. Disponível em: https://doi.org/10.5585/EccoS.n50.13998. 